\title{
Alkali Anisotropic Chemical Etching of P-Silicon Wafer
}

\author{
Marwa Nabil $^{1, a}$, Hussien. A. Motaweh ${ }^{2, b}$ \\ ${ }^{1}$ Advanced Technology and New Materials Research Institute, City for Scientific Research and \\ Technology Applications, New Borg El-Arab City, Alexandria, Egypt. \\ ${ }^{2}$ Department of Physics, Faculty of Science, Damanhour University, Egypt.

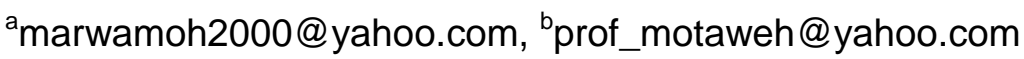

Keywords: $\mathrm{KOH}$ etchant, Anisotropy etching, Surface morphology.

\begin{abstract}
The surface chemistry of anisotropic etching of p-type Si-wafer (400) is reviewed and the anisotropic chemical etching of silicon in alkaline solution using wetting agent were discussed. The main factors which affect the production of silicon dioxide layer on crystalline silicon as a result of wet alkali anisotropic chemical etching are the concentration of etching solution $(\mathrm{KOH})$ and wetting agent (n-propanol), temperature $\left(80^{\circ} \mathrm{C}\right)$ and time of the etching $(4$ hr) process. Silicon dioxide layer has found applications in many advanced areas. The synthesized silica layer was systematically characterized by XRD, SEM and FTIR spectroscopy. The XRD results revealed the amorphous nature of silica layer. FTIR spectroscopy confirmed the presence of Si-O in produced samples. SEM confirmed the addition of n-propanol to the $\mathrm{KOH}$ solution resulted in an improvement in the etching anisotropy in a smooth etched $\mathrm{Si}$ (400) surface.
\end{abstract}

\section{Introduction}

The development of highest efficiency lowest cost solar cells requires surface conditioning steps to maximize the light trapping properties and to reduce the recombination losses on structured interfaces. The texturization of $\mathrm{Si}$ surfaces also leads to an increase in surface irregularities that result in an increase in recombination losses. It becomes critical that the damaged surface layers be removed to decrease the micro-roughness. Wet chemical processing is still the standard method used to texturize the wafer surface in solar manufacturing lines [1]. Fundamental etching techniques used in micro fabrication are dry etching (plasma phase) and wet etching (liquid phase). The disadvantages of dry etching are as follows, the gases used in dry etching are quite toxic and corrosive. It requires re-deposition of nonvolatile compounds and it needs specialized and expensive equipment [2].

Wet etching is inexpensive and it has been extensively used for the fabrication of many applications. It is the process of removing a material by using liquid chemicals or etchants from a wafer. The specific patterns are defined by masks on the wafer. Materials that are not protected by the masks are etched away by liquid chemicals. For isotropic wet etching, a mixture of hydrofluoric acid, nitric acid, and acetic acid is the most common etchant solvent for silicon [3]. When the reaction occurs, material is removed laterally at a rate similar to the speed of downward etching. Wet chemical etching is generally isotropic even though a mask is present since the liquid etchant can penetrate underneath the mask [4], as shown in Table 1. 
Table 1. Comparison between wet etching and dry etching.

\begin{tabular}{|c|c|c|}
\hline & Wet Etching & Dry Etching \\
\hline Method & Chemical Solutions & Ion Bombardment or Chemical Reactive \\
\hline $\begin{array}{l}\text { Environment and } \\
\text { Equipment }\end{array}$ & Atmosphere, Bath & Vacuum Chamber \\
\hline Advantage & $\begin{array}{l}\text { 1) Low cost, easy to implement } \\
\text { 2) High etching rate } \\
\text { 3) Good selectivity for most materials. }\end{array}$ & $\begin{array}{l}\text { 1) Capable of defining small feature size } \\
(<100 \mathrm{~nm})\end{array}$ \\
\hline Disadvantage & $\begin{array}{l}\text { 1) Inadequate for defining feature size < } \\
\text { 1 } \mu \mathrm{m} \\
\text { 2) Leads to some chemical bad effects } \\
\text { 3) Wafer Contamination issues }\end{array}$ & $\begin{array}{l}\text { 1) High cost, hard to implement } \\
\text { 2) low throughput } \\
\text { 3) Poor selectivity } \\
\text { 4) Potential radiation Damage }\end{array}$ \\
\hline Directionality & Isotropic (Except Crystalline Materials) & Anisotropic \\
\hline
\end{tabular}

Anisotropic wet etching is also known as Orientation Dependent Etching. Liquid etchants etch crystalline materials at different rates depending upon which crystal face is exposed to etchant used. A large difference in the etch rate is there depending on the silicon crystalline plane. When silicon like materials is used, this effect can allow very high anisotropy. The important factor of anisotropy etching includes selectivity, handling and process compatibility and anisotropic. Anisotropic wet etching produces a typical etch rate about $1 \mu \mathrm{m} / \mathrm{min}$.

The etchant $\mathrm{KOH}$ (Potassium Hydroxide) is used as an alternative etchant and it is the most used of all hydroxide etches. The wet anisotropic etching of silicon in $\mathrm{KOH}$ solution is a key technology for fabricating silicon microstructures [5]. Silicon etching in alkaline solutions has been employed for many years but the mechanism of the process has not been completely recognized yet, especially from the point of view of etching anisotropy, which is associated with various configurations of surface bonds [6].

The objective of this work is to form random pyramid texturing methods using new aqueous solution consisting of potassium hydroxide $(\mathrm{KOH}) / \mathrm{n}$-propanol (NPA). Reviewing surface homogeneity, pyramid size and the speed of the texturing process are compared. In this paper we describe a study of the surface morphology of Si etched in $3 \mathrm{wt} \% \mathrm{KOH}$ solutions at $80^{\circ} \mathrm{C}$.

\section{Design of the Chip Kick Mechanics}

A glass vessel supported with temperature controller and magnetic stirrer was used for the etching process. The cleaned p-type silicon (400) wafers were held vertically in the alkali etching solution. The alkaline compound used in this study is $\mathrm{KOH}(3 \mathrm{wt} \%)$ and n-propanol is used as a wetting agent (15 volume \%).

After applying a Teflon mask on one side of Si-wafer, the surface could be etched selectively. The structure and morphology were determined by XRD (X-ray 7000 Schimadzu diffractometer). The shape and random pyramid of texturization process were observed under SEM (Scanning electron microscopy, JEOL (JSM 5300)). And, the formation of chemical bonds for the texturization process of silicon-surface was determined by FTIR (Fourier Transform Infrared Spectrophotometer- Shimadzu FTIR -8400 s, Japan).

\section{Institutions Optimization Design}

With the goal to produce homogeneous surfaces which are fully covered with random pyramids of small size and little variation a wide range of different concentrations, etching times and other parameters such as preparation methods were tested. Texturized silicon-surface 
was formed in homogeneously mixed $\mathrm{KOH}$ with NPA at suitable temperature and certain time of etching.

In the beginning, a hydrogen saturated silicon surface is vertically free from attack by hydroxyl ions in $\mathrm{KOH}$ based electrolyte. If a hole reaches the surface, nucleophilic attack on a $\mathrm{Si}-\mathrm{H}$ bond by a hydroxyl group ion can occur and $\mathrm{Si}-\mathrm{OH}$ bond is formed. The $(\mathrm{Si}-\mathrm{OH})$ bond causes a polarization effect allowing a second hydroxyl group ion to attack and replace the remaining hydrogen bond. Two hydrogen atoms can then combine, injecting an electron into the substrate. The polarization induced by the $\mathrm{Si}-\mathrm{OH}$ bonds reduces the electron density of remaining $\mathrm{Si}-\mathrm{Si}$ back bonds making them susceptible to be attacked by $\mathrm{KOH}$ in a manner such that the remaining silicon surface atoms are bonded to hydrogen atoms. The silicon tetrahydroxyl molecule reacts with $\mathrm{KOH}$ to form highly stable hydroxyl anion. Then, the surface returns to its neutral state until another hole is made available [7].

\section{Test Results}

XRD detects the patterns of the p-type Si (400) wafer before and after wet-etching process. Before wet-etching, the crystal structure reveals (400) plane at $2 \theta=69.3825^{\circ}$, which is corresponding to Si-cubic structure (JCPDS Card No.01-027-1402). After 4 h of wet-etching process $\left(2 \mathrm{wt} \% \mathrm{KOH}, 15 \mathrm{vol} \% \mathrm{n}\right.$-propanol), (400) plane appears at $2 \theta=68.9806^{\circ}$ of Si structure (JCPDS Card No.00-027- 1402) accompanied with a broad peak in range $2 \theta=\left(19.9083^{\circ}\right.$ $22.8866^{\circ}$ ) corresponds to amorphous silicon dioxide (JCPDS Card No. 00-051-1594), as shown in Fig. 1.

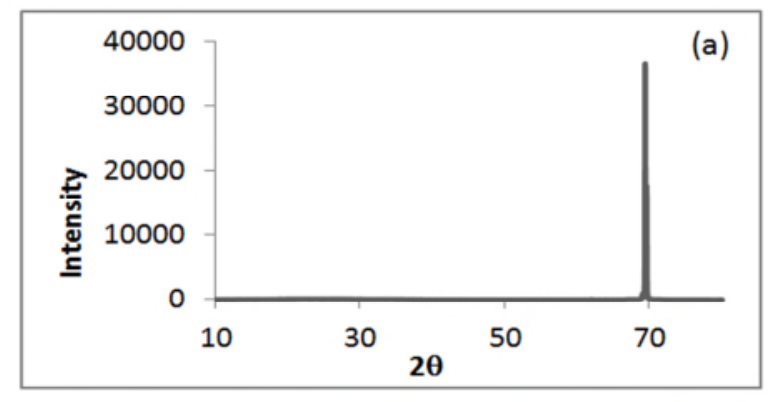

(a) Before Wet-etching

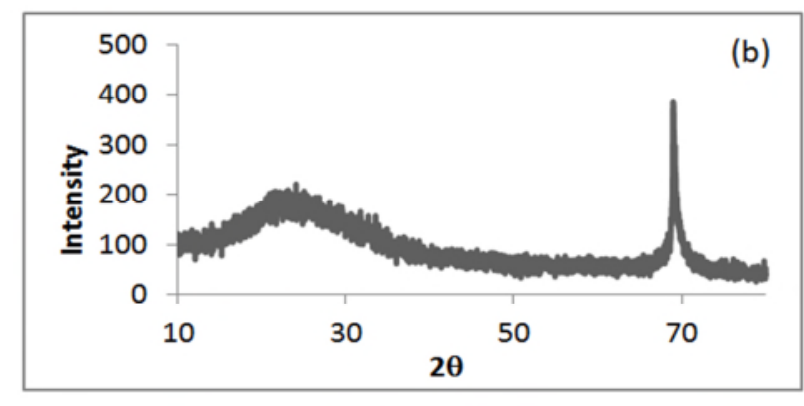

(b) After 4 hrs of Wet-etching

Fig. 1 The experimental results of XRD patterns of Si (400) wafer.

In the beginning, a hydrogen saturated silicon surface is vertically free from attack by hydroxyl ions in $\mathrm{KOH}$ based electrolyte. If a hole reaches the surface, nucleophilic attack on a $\mathrm{Si}-\mathrm{H}$ bond by a hydroxyl group ion can occur and $\mathrm{Si}-\mathrm{OH}$ bond is formed. The ( $\mathrm{Si}-\mathrm{OH})$ bond causes a polarization effect allowing a second hydroxyl group ion to attack and replace the remaining hydrogen bond. Two hydrogen atoms can then combine, injecting an electron into the substrate. The polarization induced by the $\mathrm{Si}-\mathrm{OH}$ bonds reduces the electron density of remaining $\mathrm{Si}-\mathrm{Si}$ back bonds making them susceptible to be attacked by $\mathrm{KOH}$ in a manner such that the remaining silicon surface atoms are bonded to hydrogen atoms. Then, the surface is covered by silicon dioxide layer, as shown in Fig. 2. 


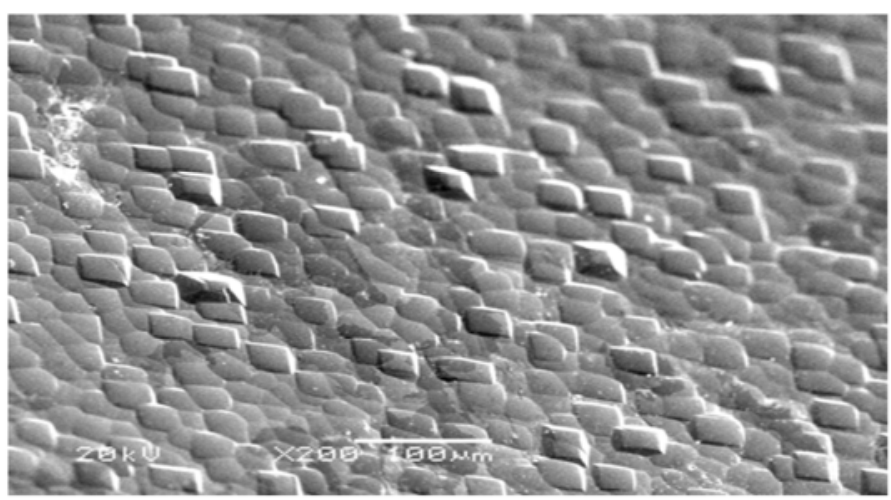

Fig. 2 The experimental results of SEM image of Si (400) wafer; after $4 \mathrm{~h}$ of wet etching in (2wt $\% \mathrm{KOH}, 15$ vol\% n-Propane), $\mathrm{T}=80^{\circ} \mathrm{C}$.

Then, in case of $\mathrm{KOH}$, the optimum temperature at which the etching process is carried out using this etchant is $80^{\circ} \mathrm{C}$. This is because the optimum etching rate with minimum surface roughness is obtained in this condition. The etched silicon surface smoothens with both increase in $\mathrm{KOH}$ concentration and bath temperature. Silicon surface roughness degrades with increase in etch duration due to the masking of hydrogen bubbles evolved during etching which significantly contributes to surface roughness.

Potassium hydroxide $(\mathrm{KOH})$ is an alkaline solution, normally used as the etchant for the anisotropic wet process. The former has an excellent uniformity and reproducibility, but notcompatible with an electrical circuits. Generally, the usage of $\mathrm{KOH}$ becomes the best choice in the case of that the engineers simply produce the micro-structures onto the Si wafer. The etching rate by $\mathrm{KOH}$ strongly depends on the crystallographic orientations of the Si material. The overall chemical etching reaction by alkaline solution is given by, as shown in Eq. 1: [8].

$$
\mathrm{Si}+2 \mathrm{OH}^{-}+2 \mathrm{H}_{2} \mathrm{O}--->\mathrm{SiO}_{2}(\mathrm{OH})_{2}^{-2}+2 \mathrm{H}_{2}
$$

Silicon reacts with water and an $\mathrm{OH}$ - ion and produces hydroxide ion and hydrogen gas bubbles. The dependency of the etching rate on the crystallographic orientation said to be the differences of the number of dangling bond at the surfaces. The chemical mechanism behind it is removal of silicon atom in $\mathrm{KOH}$ solution takes place in two steps: First, four electrons are affected in bulk silicon, as shown in Eq. 2.

$\mathrm{Si} .+4(\mathrm{OH})------>\mathrm{Si}(\mathrm{OH}) 4 .+4 \mathrm{e}-$

In second step, the electrons are released back into the solution accordingly, as shown in Eq. 3.

$$
\text { 4e.-+ 4H2O -----> 4(OH).- +2 } \mathrm{H} 2
$$

Products in first step $\mathrm{Si}(\mathrm{OH})_{4}$, is supposed to soluble in water. But actually, $\mathrm{Si}(\mathrm{OH})_{4}$ is decompose into water and silicon-dioxide. The probability of removal of particular silicon atom depends on temperature and microscopic activation energy.

FTIR indicates (Fig. 3) the broad peak at (3000-3700) $\mathrm{cm}^{-1}$, which indicated stretching of O$\mathrm{H}$ bond in $\mathrm{SiOH}$ group. Band in region (1000-1200) $\mathrm{cm}^{-1}$ is assigned to $\mathrm{Si}-\mathrm{O}$ a symmetric stretching in Si-O-Si. Noticeable, disappearing of peaks in region (1400-1600) $\mathrm{cm}^{-1}$, in (b), which corresponds to mix of stretching mode $\mathrm{Si}-\mathrm{Si}$ and wagging mode $\mathrm{Si}-\mathrm{Hn}(\mathrm{n}=1$ and 2). So, the best time of etching process is $4 \mathrm{~h}$, for formation silica layer on $\mathrm{Si}$-wafer surface. 


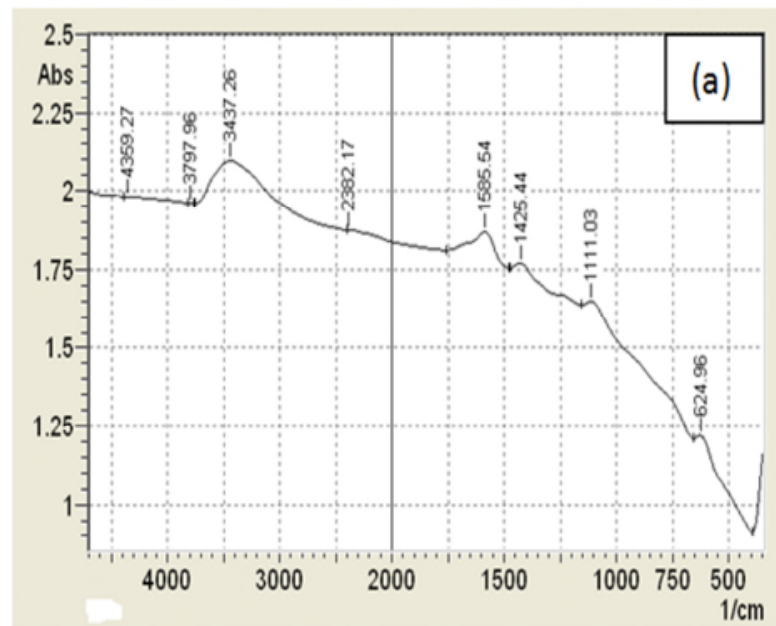

(a) Before wet-etching process

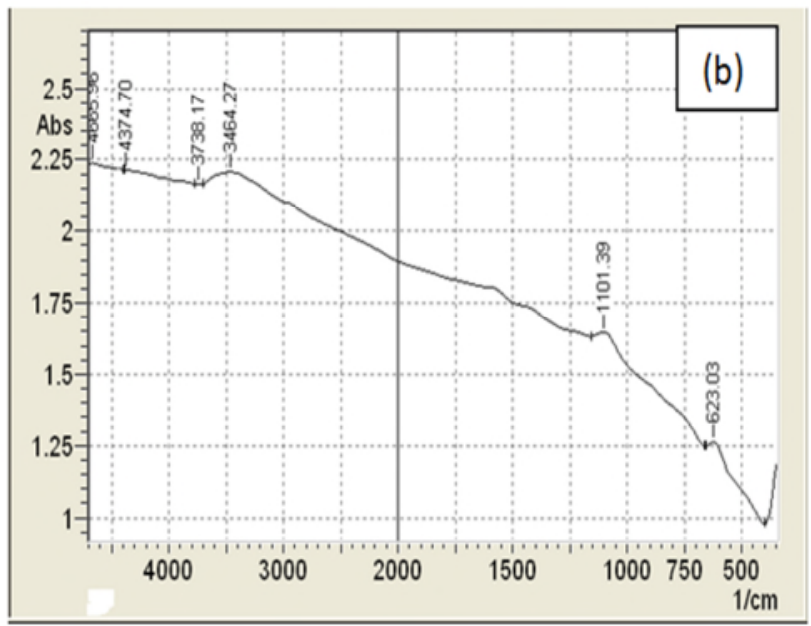

(b) After $4 \mathrm{~h}$ of wet-etching process

Fig. 3 The experimental results of FTIR Spectra of Si (400) wafer.

\section{Conclusion}

The present work is a study of the preparation of silicon dioxide layers on p-type crystalline silicon wafers (400) and a discussion of the obtained samples. The process of wet alkali anisotropic chemical etching process was monitored as the effect of concentration of alkaline etching solution $(\mathrm{KOH})$, concentration of wetting agent (n-propanol), temperature and time of etching process. XRD spectrum showed amorphous peak of silicon dioxide layer. This particular property might be useful for decreasing the light scattered for optoelectronic devices. Finally, the peculiar hollow structure of the silicon dioxide nano particles could also be useful for trapping or storing molecules. From the FTIR data, it is shown that all samples exhibited characteristic peaks for silicon dioxide.

\section{Acknowledgement}

In this paper, the research was sponsored by (SRTA-City), City of Scientific Research \& Technological Applications, New Borg El-Arab City, Alexandria, Egypt.

\section{References}

[1] K.abd. Intessar, K.al-Bity. Abtisam, A. Esmael. Ahmed, A. Bayat. Salah. Chemical etching of Si-p-type wafers using KOH. Diyala J. Pure Sci. 8(4) (2012) 10-15.

[2] J. Benjamin, J. Grace Jency, G. Vijila. A review of different etching methodologies and impact of various etchants in wet etching in micro fabrication. Int. J. Innov. Res. Sci., Eng. Tech. 3 (2014) 558-564.

[3] K. Biswas, S. Kal. Etch characteristics of KOH, TMAH and dual doped TMAH for bulk micromachining of silicon. Microelec. J. 37(6) (2006) 519-525.

[4] C. R. Yang, P. Y. Chen, C. H. Yang, Y. C. Chiou, R. T. Lee. Effects of various ion-typed surfactants on silicon anisotropic etching properties in $\mathrm{KOH}$ and TMAH solutions. Sensor. Actuat. A. 119(1) (2005) 271-281. 
[5] H. Tanaka, S. Yamashita, Y. Abe, M. Shikida, K. Sato. Fast etching of silicon with a smooth surface in high temperature ranges near the boiling point of $\mathrm{KOH}$ solution. Sensor. Actuat. A. 114(2-3) (2004) 516-520.

[6] I. Zubel, K. Rola, M. łgorzata Kramkowska. The effect of isopropyl alcohol concentration on the etching process of Si-substrates in KOH solutions. Sensor. Actuat. A. 171(2) (2011) 436445.

[7] A. Hady Kashyout, H. M. A. Soliman, M. Nabil, A. A. Bishara. Fabrication of nano-porous silicon using alkali etching process. Mater. Lett. 100 (2013) 184-187.

[8] H. Seidel, et al. Anisotropic etching of crystalline silicon in alkaline solutions, Orientation dependence and behavior of passivation layers. J. Electrochem. Soc. 137(11) (1990) 3612-3626. 